CYSTIC FIBROSIS

\title{
Diagnostic and prognostic value of serum antibodies against Pseudomonas aeruginosa in cystic fibrosis
}

\author{
M Kappler, A Kraxner, D Reinhardt, B Ganster, M Griese, T Lang
}

See end of article for authors' affiliations

\section{Correspondence to:} Professor DrM Griese, von Haunersches Kinderspital, Ludwig-Maximilians-

University, Lindwurmstr 4, 80337 Munich, Germany; Matthias.Griese@med.unimuenchen.de

Received 17 July 2005

Accepted

20 December 2005

Published Online First

31 January 2006
Background: Eradication of Pseudomonas aeruginosa in patients with cystic fibrosis (CF) is possible if initiated early in the course of colonisation. To detect $P$ aeruginosa as early as possible is therefore a major goal. This study was undertaken to validate a commercialised test for the detection of serum Pseudomonas antibodies in patients with $\mathrm{CF}$.

Methods: A representative cross sectional analysis of serum antibodies against three Pseudomonas antigens (alkaline protease, elastase, and exotoxin A) was performed in 183 patients with CF of mean age 16.7 years and $\mathrm{FEV}_{1} 85.9 \%$ predicted. The results were correlated with microbiological results from the previous 2 years to calculate sensitivity, specificity, positive and negative predictive values. The following 2 years were assessed to determine prognostic predictive values.

Results: A combination of all three tested antibodies yielded the best results with a sensitivity of $86 \%$, specificity of $96 \%$, and a positive predictive value of $97 \%$. These values were higher if only patients in whom sputum cultures were available were considered $(n=76$, sensitivity $95 \%$, specificity $100 \%$, positive predictive value $100 \%)$. The prognostic positive predictive value was high in intermittently infected patients $(83 \%)$ but low in patients free of infection $(33 \%)$, whereas the prognostic negative predictive value was high in patients free of infection (78\%) and low in intermittently infected patients (58\%).

Conclusions: Regular determination of serum antibodies may be useful in CF patients with negative or intermittent but not with positive $P$ aeruginosa status. A rise in antibody titres indicates probable infection and eradication treatment may be initiated even in the absence of microbiological detection of $P$ aeruginosa.
$C$ stic fibrosis (CF) is the most frequent life threatening autosomal recessive disorder in white subjects. It is caused by mutations in the cystic fibrosis transmembrane conductance regulator (CFTR) gene, leading to disturbed ion transport across epithelial cells. The clinical consequences of this basic defect are seen in exocrine glands, causing pancreatic insufficiency and progressive pulmonary insufficiency which is the major cause of morbidity and mortality in CF patients. The airway epithelium is susceptible to lower respiratory tract colonisation involving infection and inflammation, even in patients with clinically mild lung disease. ${ }^{1}$ The most frequently reported respiratory pathogen is Pseudomonas aeruginosa, ${ }^{2}$ which is cultured in specimens from up to $21 \%$ of CF children under 1 year of age and, in the absence of a policy of early eradication treatment, increasing to $>80 \%$ at 26 years or older. ${ }^{23}$ Identification of mucoid forms of $P$ aeruginosa is thereby an unfavourable prognostic factor for survival. ${ }^{4}$

Eradication of this organism is usually not possible in cases of chronic colonisation and infection, but there is evidence that early antibiotic treatment reduces the rate of positive cultures in CF patients with newly isolated $P$ aeruginosa. ${ }^{5}$ A major goal is therefore to detect $P$ aeruginosa as early as possible to use the window of opportunity for possible eradication. ${ }^{6}$ From this point of view, bronchoalveolar lavage or spontaneous or induced sputum cultures obtained at regular intervals would be the desirable "gold standard" methods for the microbiological detection of $P$ aeruginosa. ${ }^{7}$ However, in clinical practice we often have to rely on the results from oropharyngeal swab cultures because the patients are too young or too healthy to expectorate, and this method is the only direct and readily noninvasive technique available to obtain respiratory secretions for culture.

An alternative approach is to track $P$ aeruginosa colonisation by testing for serum antibodies against this organism, especially as they may be detected clearly before the organism is isolated from respiratory samples. ${ }^{28}$ Since serum precipitins and antibodies against $P$ aeruginosa have been described $^{2-11}$ and the first ELISA against Pseudomonas antigens was developed, ${ }^{12}$ attempts have been made to correlate the acquisition of $P$ aeruginosa with the production of an antibody response against this organism. ${ }^{13-19}$ Reviewing the published data, a correlation between microbiological findings, clinical state, and results of antibody determination is definite for cohorts of CF patients, whereas the impact of an individual result of serum antibodies against $P$ aeruginosa remains difficult to interpret.

This study was undertaken to assess the diagnostic accuracy of a commercial antibody ELISA test in relation to the microbiological findings from respiratory secretions and to estimate the prognostic value of these antibody test results to anticipate the future trend of microbiological results. The particular test is a further development of a radioimmunoassay developed by Döring and Hoiby ${ }^{18}$ which permits the determination of three major extracellular proteins of $P$ aeruginosa-namely, alkaline protease (AP), elastase (E), and exotoxin A (EA). These proteins show high grade immunogenicity and are expressed by nearly all strains of $P$ aeruginosa.

\section{METHODS}

Subjects and study design

Routine regular determination of serum antibodies against $P$ aeruginosa was introduced in our CF centre in 2000. To conduct a representative cross sectional analysis of $P$ aeruginosa antibody titres in patients with $\mathrm{CF}$, antibody test

Abbreviations: $A P$, alkaline protease; $C F$, cystic fibrosis; $E$, elastase; EA, exotoxin A; ELISA, enzyme linked immunoassay; $F V_{1}$, forced expiratory volume in 1 second; NPV, negative predictive value; OD, optical density; PPV, positive predictive value 
Table 1 Clinical data of 183 patients with CF according to their $P$ aeruginosa status

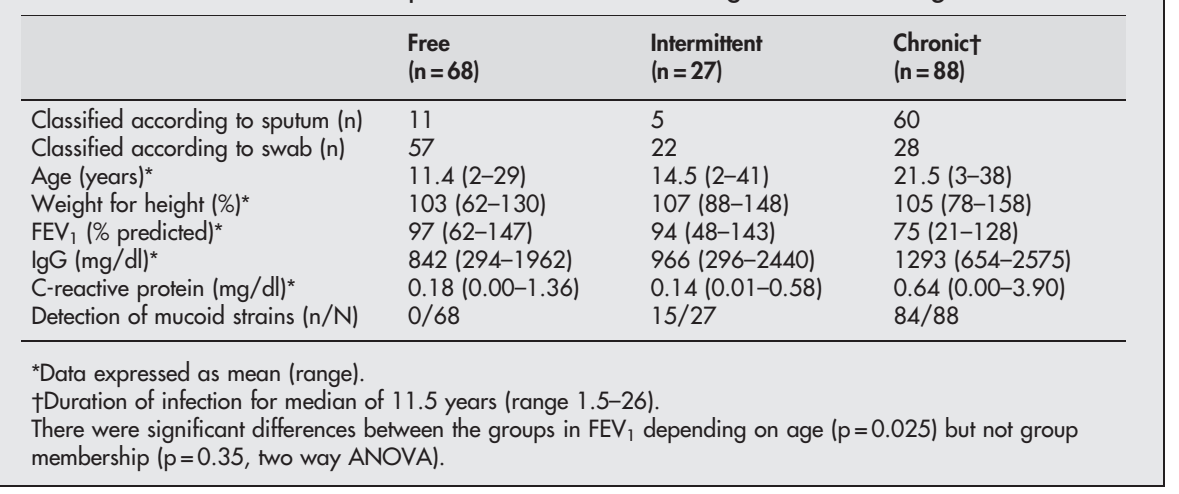

results obtained between 2000 and 2002 were extracted. The antibody test results were correlated, firstly, with known microbiological data from the previous 2 years and, secondly, with microbiological results from the following 2 years.

Of 421 patients with CF attending the Cystic Fibrosis Centre at the Children's University Hospital in Munich between 2000 and 2002 (mean age 16.3 years, median 15.4, range $0.4-41), 212$ patients were seen continuously at three monthly intervals by one physician of whom 187 agreed to participate in the study. Three patients were excluded from the study because, after vaccination against $P$ aeruginosa, they had known serum antibodies against EA. One patient was excluded because, after 14 years of chronic $P$ aeruginosa infection, he underwent lung transplantation 3 years before the study was started and consequently changed microbiologically to not being infected with this organism. The mean age of the remaining 183 patients was 16.7 years (range 15.7- 24.1), mean forced expiratory volume in 1 second $\left(\mathrm{FEV}_{\mathrm{l}}\right)$ was $85.9 \%$ predicted (median 89; range 20-147), mean weight for height 105\% (median 104; range 62-158). 181 patients $(99 \%)$ were on continuous (>300 days/year) oral antibiotic therapy against Staphylococcus aureus. Weight and height were measured at the beginning of the study and the weight for height predicted was calculated using height percentile standards given by Prader et al. ${ }^{20}$

The patients were divided into three groups according to the number of positive microbiological cultures of $P$ aeruginosa in the previous 2 years: free of $P$ aeruginosa ( 0 positive of 8 cultures); intermittently colonised ( $1-6$ positive of 8 cultures); and chronically colonised ( $\geqslant 7$ positive of 8 cultures). The clinical details of these subjects are shown in table 1 and their age distribution and $P$ aeruginosa status are given in fig 1 .

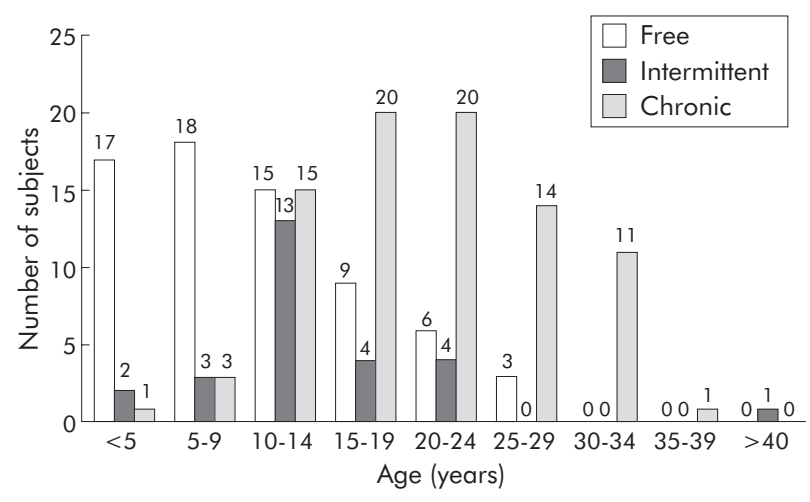

Figure 1 Age distribution of 183 patients with CF divided into three groups according to their microbiological $P$ aeruginosa status: free of $P$ aeruginosa (0 positive of 8 cultures); intermittently colonised (1-6 positive of 8 cultures); and chronically colonised ( $\geqslant 7$ positive of 8 cultures).
Complete microbiological data over the following 2 years for at least seven of eight possible microbiological samples was available in 162 of the 183 patients (overall rate $88.5 \%, 66 / 68$ patients in the $P$ aeruginosa free group, $24 / 27$ patients in the intermittently infected group, and $68 / 88$ patients in the chronically infected group).

Informed consent was obtained from all subjects. The study was approved by the local institutional review board for human studies of the University Children's Hospital, Munich.

\section{Microbiological and serological assays}

Sputum samples or deep oropharyngeal swabs were routinely obtained every 3 months and, after homogenisation, were cultured on blood agar plates and McConkey agar plates for at least 72 hours aerobically at $37^{\circ} \mathrm{C}$ without the use of additional selective media for $P$ aeruginosa.

Antibody titres against the three purified $P$ aeruginosa antigens (AP, E, and EA) were determined using a commercially available ELISA test system (Mediagnost, Germany) according to the manufacturer's instructions. Two replicates were used for each sample. The antibody titres were expressed in arbitrary units and categorised as 0 (titre negative $<1: 500$ ) or 1 (titre positive $>1: 500$ ). The calibration curves of the test system were linear for optical density (OD) values between 0.1 and 1.7, corresponding to titres of $1: 10$ to $1: 2000$. Negative and positive controls were provided by the manufacturer and consisted of pooled serum samples from $P$ aeruginosa positive CF patients and from normal children, respectively. All measurements were conducted in duplicate for each ELISA panel. For determination of high titres $(>2000)$, dilutions of 1:10 to 1:100 were used. The cut off, depending on the slope of the calibration curve, was reached at a mean (SD) OD of $0.35(0.046)$ for all three antibodies.

\section{Intra- and inter-assay variability of serological assays}

The intra-assay variability (correlation between the results of duplicate measurements) was excellent for the three different antibodies with a Spearman coefficient of correlation of $0.988-0.995$ (100\% correlation is 1.000$)$ and a Bland-Altman coefficient of repeatability of $0.091-0.096$ ( $100 \%$ correlation is $0.000)$.

The inter-assay variability (correlation between the results obtained to generate the ELISA calibration curves) was satisfactory with a relative coefficient of variation of 7.3-20.3 (a coefficient of 0 represents complete concordance and 100 represents no agreement).

\section{Statistical analysis}

The sensitivity, specificity, 95\% confidence intervals (95\% CI), positive predictive value (PPV), and negative predictive value 
Table 2 Anti- $P$ aeruginosa antibodies in patients with CF correlated with $P$ aeruginosa status defined retrospectively

\begin{tabular}{|c|c|c|c|c|c|c|}
\hline \multirow[b]{2}{*}{ Antibodies against } & \multicolumn{3}{|c|}{$P$ aeruginosa status according to culture results } & \multicolumn{2}{|c|}{ Comparison* ( $p$ values) } & \multirow[b]{2}{*}{$\mathrm{CvF}$} \\
\hline & $\begin{array}{l}\text { Free (F) } \\
(\mathrm{N}=68)\end{array}$ & $\begin{array}{l}\text { Intermittent (I) } \\
(\mathrm{N}=27)\end{array}$ & $\begin{array}{l}\text { Chronic (C) } \\
(\mathrm{N}=88)\end{array}$ & F vI & $\mathrm{I} v \mathrm{C}$ & \\
\hline $\begin{array}{l}\text { Alkaline protease (AP) } \\
\text { Frequency of antibody positive (n/N) }\end{array}$ & $1 / 68$ & $7 / 27$ & $66 / 88$ & $<0.001$ & $<0.001$ & $<0.001$ \\
\hline $\begin{array}{l}\text { Elastase }(E) \\
\text { Frequency of antibody positive }(n / N)\end{array}$ & $0 / 68$ & $4 / 27$ & $57 / 88$ & $<0.001$ & $<0.001$ & $<0.001$ \\
\hline $\begin{array}{l}\text { Exotoxin } A(E A) \\
\text { Frequency of antibody positive }(n / N)\end{array}$ & $3 / 68$ & $11 / 27$ & $73 / 88$ & $<0.001$ & $<0.001$ & $<0.001$ \\
\hline $\begin{array}{l}\text { Cumulative score (AP/E/EA) } \\
\text { Frequency of antibody score } \geqslant 1(n / N)\end{array}$ & $3 / 68$ & $13 / 27$ & $86 / 88$ & $<0.001$ & $<0.001$ & $<0.001$ \\
\hline
\end{tabular}

Antibody titres were scored as 0 (titre negative $<1: 500$ ) and 1 (titre positive $>1: 500$ ).

*Mann-Whitney test for comparison of antibody levels and Fisher's exact test for comparison of frequency of patients with antibody score $\geqslant 1$, corrected for multiple comparisons by Bonferroni method.

(NPV) were calculated from contingency tables with confidence intervals calculated using the binominal exact method.

To briefly review the concept of diagnostic accuracy with respect to serum antibodies against $P$ aeruginosa, sensitivity is the probability that a patient who is colonised with $P$ aeruginosa shows detectable antibodies against this organism, and specificity is the probability that a patient who is not colonised with $P$ aeruginosa shows no detectable antibodies against this organism. The sensitivity and specificity are properties of the test and are not determined by the prevalence of $P$ aeruginosa colonisation in the population studied. Therefore, neither the sensitivity nor the specificity answer the most important questions-namely, if the antibody result is positive, what is the chance that the patient is really colonised with $P$ aeruginosa, and if the antibody result is negative, what is the chance that the patient is not colonised with this organism? The answers to these questions are given by the predictive values which take into account the prevalence: the PPV is the probability that a subject with positive antibodies against $P$ aeruginosa is colonised with that organism; the NPV is the probability that a subject with a negative antibody result is not colonised with $P$ aeruginosa.

In this study we calculated the predictive values accounting for the $P$ aeruginosa prevalence retrospectively determined in our study population according to microbiological results. Furthermore, we calculated the prospective predictive values using the antibody test results and the microbiological results of the following 2 years. The later values state the probability for a single CF patient to shift his or her $P$ aeruginosa status dependent on his or her initial antibody titre.

For comparison of paired data, the Spearman coefficient of correlation and the relative coefficient of variation were calculated and the coefficient of repeatability was calculated according to the method proposed by Bland and Altman. ${ }^{21}$ The Mann-Whitney test was used for comparison of unrelated data and Fisher's exact test was used for categorical variables, corrected for multiple comparisons by the Bonferroni method. Analyses were performed using SPSS version 12.0 for Windows (SPSS Inc, Chicago, IL, USA). Data are given as mean (median, range).

\section{RESULTS}

\section{Serum antibodies against $P$ aeruginosa and correlation with $P$ aeruginosa status defined retrospectively}

The levels and positivity of the three antibodies (AP, E, and EA) differed substantially between the three groups of $\mathrm{CF}$ patients (table 1). A negative single test was scored as 0 and a positive test as 1 , thus giving a cumulative score (AP or E or EA) from 0 to a maximum of 3 . The patients free of infection, as determined by culture results from the previous 2 years, had positive antibody results in only a very small fraction (3/ 68 patients) and the mean cumulative score was low (0.06). The patients with chronic infection had a very high rate of positive antibody results (86/88 patients) and the mean cumulative score was high (2.22). The patients with intermittent infection were in between (13/27 patients positive) with a mean cumulative score of 0.81 (table 2 ).

\section{Sensitivity and specificity of serum antibodies against $\boldsymbol{P}$ aeruginosa compared with $\boldsymbol{P}$ aeruginosa status defined retrospectively}

To calculate sensitivity and specificity from the above data it was necessary to categorise intermittently infected patients as either negative or as positive. Since $P$ aeruginosa had been detected at least once, they were all defined as positive. Thus, two groups of patients were defined: 68 patients who were free of $P$ aeruginosa and 115 patients in whom $P$ aeruginosa had been detected within the previous 2 years.

Overall, specificity was satisfactory (AP 98.5, E 100, EA 95.6) but sensitivity partially was very low (AP 63.5, E 53.0, EA 73.0). The results for sensitivity were better if only patients with sputum cultures were considered (AP 76.9, E 67.7, EA 78.5) and were worse if only patients with swabs were considered (AP 48.0, E 34.0, EA 66.0). The results for specificity were excellent if only patients with sputum cultures were considered (AP 100, E 100, EA 100) and were only slightly worse if only patients with swabs were considered (AP 98.2, E 100, EA 94.7).

The combination of antibody results by counting exclusively multiple positive results as positive $(\mathrm{AP}+\mathrm{E}, \mathrm{AP}+\mathrm{EA}$, $\mathrm{E}+\mathrm{EA}, \mathrm{AP}+\mathrm{E}+\mathrm{EA})$ generated unsatisfactory results for sensitivity (41.7-52.2\%) but good results for specificity (98.6$100 \%)$. The combination of antibody results by assessing a patient as positive with one of two results positive (AP/E, AP/ EA, E/EA) generated somewhat better results for sensitivity (70.4-84.3\%) but worse results for specificity (90.3-97.2\%).

A combination of all three antibodies (AP/E/EA, cumulative antibody score) yielded the best results. Patients were counted as positive if any of the three antibodies was positive and negative if no positive antibody result was obtained (table 3).

\section{Prognostic value of antibody results}

Of the 183 patients, a cohort of 162 patients was studied at three monthly intervals for microbiological status over a period of 2 years following first antibody determination. This 
Table 3 Sensitivity and specificity of cumulative anti- $P$ aeruginosa antibody scores according to the method by which material was obtained for microbiological culture

\begin{tabular}{llll}
\hline & Sputum only & All (sputum + swab) & Swab only \\
\hline Number of subjects (n) & 76 & 183 & 107 \\
Sensitivity \% (95\% Cl) & $95.4(87.1$ to 99.0$)$ & $86.1(78.4$ to 91.8$)$ & $74.0(59.7$ to 85.4$)$ \\
(n/N) & $62 / 65$ & $99 / 115$ & $37 / 50$ \\
Specificity \% (95\% Cl) & $100(71.5$ to 100$)$ & $95.6(87.6$ to 99.1$)$ & $94.7(85.4$ to 98.9$)$ \\
(n/N) & $11 / 11$ & $65 / 68$ & $54 / 57$ \\
PPV\% (95\% Cl) & $100(94.2$ to 100$)$ & $97.1(91.6$ to 99.4$)$ & $92.5(79.6$ to 98.4$)$ \\
NPV \% (95\% Cl) & $78.6(49.2$ to 95.3$)$ & $80.2(69.9$ to 88.3$)$ & $80.6(69.1$ to 89.2$)$ \\
\hline
\end{tabular}

PPV, positive predictive value; NPV, negative predictive value.

Predictive values were calculated for the given prevalence-that is, $61.5 \%$ for all patients $(115 / 183), 85.5 \%$ for patients with sputum cultures $(65 / 76)$, and $45.0 \%$ for patients with swabs $(50 / 107)$.

allowed the prognostic values of the antibody determinations to be calculated separately for patients free of $P$ aeruginosa and for those with intermittent $P$ aeruginosa colonisation. Of 63 patients free of $P$ aeruginosa and with negative antibody results, 14 patients became intermittently infected during the following 2 years and 49 patients remained free of $P$ aeruginosa. Of three patients free of $P$ aeruginosa but with positive antibody results, one patient changed to intermittent infection and two patients remained free of $P$ aeruginosa. In the intermittently infected group of 24 patients, 12 patients had no antibodies. Microbiological follow up was negative in nine of them and positive in three. Of the other 12 patients with positive serum antibodies, two became free of $P$ aeruginosa within the following 2 years and 10 remained intermittently infected.

The prognostic PPV was therefore low in patients free of $P$ aeruginosa $(33.3 \%)$ and high in intermittently infected patients $(83 \%)$. The prognostic NPV was high in patients free of $P$ aeruginosa $(77.8 \%)$ and $58 \%$ in patients with intermittent infection (table 4).

\section{DISCUSSION}

The first part of our study indicated a close correlation between the microbiologically defined status of $P$ aeruginosa and serum antibodies against this organism in patients with $\mathrm{CF}$, as high values for sensitivity and specificity of serum antibodies were obtained. This confirms similar observations described by others. ${ }^{13-19}$ However, the clinical relevance of a test can best be derived from the positive and negative predictive values which, in our case, showed that a positive antibody result indicated the presence of $P$ aeruginosa in the airways of a CF patient with $>93 \%$ probability. For patients with CF chronically infected with $P$ aeruginosa and positive antibody test results, routine follow up antibody determinations are therefore of no use. On the other hand, a negative antibody result made the presence of $P$ aeruginosa unlikely $(80 \%)$.

Table 4 Prognostic values of serum antibody determination: results from evaluation over the 2 years following the initial determination of antibody titres

\begin{tabular}{lll}
\hline & Free $(\mathrm{N}=66)$ & Intermittent $(\mathrm{N}=24)$ \\
\hline PPV \% (95\% Cl) & $33.3(0.8$ to 90.6$)$ & $83.0(51.6$ to 97.9$)$ \\
$\mathrm{n} / \mathrm{N}$ & $1 / 3$ & $10 / 12$ \\
$\mathrm{NPV} \%(95 \% \mathrm{Cl})$ & $77.8(76.5$ to 94.4$)$ & $58.0(27.7$ to 84.8$)$ \\
$\mathrm{n} / \mathrm{N}$ & $49 / 63$ & $7 / 12$ \\
\hline
\end{tabular}

PPV, positive predictive value; NPV, negative predictive value Predictive values were calculated on the basis of the prevalence of $P$ aeruginosa after 2 years based on the serum antibody results $(15 / 66$ $(22.7 \%)$ in the $P$ aeruginosa free group and $13 / 24(54.2 \%)$ in the intermittently infected group) to determine the probability for a CF patient to shift his or her $P$ aeruginosa status depending on the antibody titre.
In the second part of our study we tested the prognostic predictive values of antibody determinations, assessing the time period 2 years after initial antibody determination. Unexpectedly, the prognostic PPV was low for patients who were not infected with $P$ aeruginosa (table 4 ). Although the number of patients in this study is too small for this value to have any significance, we have tried to explain this surprising finding. Of three patients with positive antibody results despite negative retrospective microbiology, only one patient became microbiologically positive during the following 2 years but the other two remained $P$ aeruginosa free. This finding was not consistent with the very high PPV calculated from the first observation period. Detailed analysis of the two patients with the unexpected positive antibody titres provided possible clues: one patient had been colonised with $P$ aeruginosa 5 years before our study for 18 months and the bacteria were successfully eradicated. The other patient was put on inhaled antipseudomonal therapy using tobramycin for 4 years before our study because of initial clinical instability without any microbiologically detected $P$ aeruginosa. His subsequent clinical improvement might reflect successful eradication of $P$ aeruginosa, which had been present in the lungs and had escaped microbiological detection.

The prognostic NPV in the group free of $P$ aeruginosa was $78 \%$, suggesting that, in patients with negative antibody results, the probability of $P$ aeruginosa being detected within the next 2 years was around $20 \%$ ( $10 \%$ per year). This value is in the order of the annual rate of new infections of about $10 \%$ per year. ${ }^{22}$ The prognostic NPV of the test was therefore excellent.

The prognostic predictive values calculated for the group of intermittently infected patients can be applied to patients in whom $P$ aeruginosa is detected for the first time in swab or sputum culture. If the serum antibodies were positive, it was very likely ( $83 \%$ ) that the patient maintained the $P$ aeruginosa infection; if the serum antibodies were negative, the chance of elimination of $P$ aeruginosa was $58 \%$ (table 4 ).

The microbiological status of a patient in our study was retrospectively defined by sputum samples or oropharyngeal swabs taken regularly every 3 months. According to this, we cannot differentiate between colonisation and infection since we believe these two conditions to be a continuum in patients with CF. Although this widespread regimen is feasible in everyday patient care and approximates the recommendations for surveillance of CF patients, ${ }^{23}$ the results of swab analyses may be erroneous ${ }^{24}$ and the impact of swab results in previous studies lead to controversial conclusions, yielding either a high PPV ${ }^{25}$ or a high NPV. ${ }^{26}{ }^{27}$ Partly relying on swab culture results may thus introduce some inaccuracy into the interpretation of our serological data. We therefore considered sputum or swab culture results separately. For patients in whom sputum cultures were available, the PPV of the cumulative antibody test results was $100 \%$, meaning that a CF patient with positive results in any antibody determination 
actually has been colonised with $P$ aeruginosa within the previous 2 years. As sputum is considered the reference method, the same conclusion must be drawn for patients in whom only oropharyngeal swabs were available. This means that, independent of the method by which material for microbiological testing was obtained, a positive cumulative serum antibody result indicates $P$ aeruginosa colonisation within the previous 2 years.

Interestingly, the NPV of the cumulative antibody results of around $80 \%$ did not change if sputum or swab culture results were calculated independently, indicating that negative results of antibody determination did not rule out future colonisation with $P$ aeruginosa.

Our calculations of PPV and NPV depend on the prevalence of $P$ aeruginosa and thereby on successful early therapeutic intervention strategies and on the age distribution of our cohort, so our conclusions may not be applicable to other populations of CF patients. Overall, the prevalence of $P$ aeruginosa in our patients $(61.5 \%)$ was high and comparable to published data. ${ }^{28}$ Among adult patients the rate of chronic $P$ aeruginosa colonisation was $67 \%$, which is comparable to data from adult CF centres showing a chronic $P$ aeruginosa infection rate of about $70 \%{ }^{22}$ The rate of $P$ aeruginosa detection in children under the age of 6 years was $18 \%$ in our group, which is lower than the $30 \%$ reported elsewhere. ${ }^{29}$

As the predictive values depend on the prevalence of $P$ aeruginosa, approximate adjustments need to be made for individual patients. In younger patients the prevalence of $P$ aeruginosa is lower, leading to a worsening of the PPV but to an improvement in the NPV. Using our results for the sensitivity (86\%) and specificity (90\%) of the test, the NPV is only $80 \%$ for the $P$ aeruginosa prevalence of $61 \%$ in our study group compared with $96 \%$ for the prevalence of $18 \%$ found in children less than 6 years of age. This means that negative serum antibodies against $P$ aeruginosa make an infection with this organism less likely, the younger the child is.

The determination of a collection of three serum antibodies against $P$ aeruginosa (AP, E, and EA) in patients with $\mathrm{CF}$ is reliable if cumulative antibody scores are used. Positive antibody results almost prove colonisation with $P$ aeruginosa, while negative test results indicate the absence of $P$ aeruginosa with increasing probability as age decreases. We conclude that, from a clinical perspective, regular determination of serum antibodies against $P$ aeruginosa may be useful in patients with negative microbiological $P$ aeruginosa status. If antibody titres rise under these conditions, there is a high suspicion for possible infection with $P$ aeruginosa and we suggest using eradication treatment, even in the absence of microbiological detection of $P$ aeruginosa. At present our regime for eradication of $P$ aeruginosa relies solely on the microbiological detection of the organism. In future, early initiation and possibly the intensity and duration of antipseudomonal treatment may be determined by antibody test results, but more longitudinal data will be necessary to achieve this.

\section{Authors' affiliations \\ M Kappler, A Kraxner, D Reinhardt, B Ganster, M Griese, T Lang, \\ Children's University Hospital of the Ludwig-Maximilians-University Munich, Germany \\ Funding: none. \\ Competing interests: none.}

These data are part of the medical thesis of Angelika Kraxner. Parts of the study have been presented in abstract form during the annual North American Cystic Fibrosis Conference in Los Angeles, 2003.

\section{REFERENCES}

1 Konstan MW, Hilliard KA, Norvell TM, et al. Bronchoalveolar lavage findings in cystic fibrosis patients with stable, clinically mild lung disease suggest ongoing infection and inflammation. Am J Respir Crit Care Med 1994:150:448-54.

2 Pier GB. Role of the cystic fibrosis transmembrane conductance regulator in innate immunity to Pseudomonas aeruginosa infections. Proc Natl Acad Sci USA 2000;97:8822-8.

3 FitzSimmons SC. The changing epidemiology of cystic fibrosis. J Pediatr 1993; 122:1-9.

4 Henry RL, Mellis CM, Petrovic L. Mucoid Pseudomonas aeruginosa is a marker of poor survival in cystic fibrosis. Pediatr Pulmonol 1992;12:158-61.

5 Marchetti F, Giglio L, Candusso M, et al. Early antibiotic treatment of pseudomonas aeruginosa colonisation in cystic fibrosis: a critical review of the literature. Eur J Clin Pharmacol 2004;60:67-74.

6 Li Z, Kosorok MR, Farrell PM, et al. Longitudinal development of mucoid Pseudomonas aeruginosa infection and lung disease progression in children with cystic fibrosis. JAMA 2005;293:581-8.

7 Thomassen MJ, Klinger JD, Badger SJ, et al. Cultures of thoracotomy specimens confirm usefulness of sputum cultures in cystic fibrosis. J Pediatr 1984; 104:352-6

8 West SE, Zeng L, Lee BL, et al. Respiratory infections with Pseudomonas aeruginosa in children with cystic fibrosis: early detection by serology and assessment of risk factors. JAMA 2002;287:2958-67.

9 Hoiby N, Wiik A. Antibacterial precipitins and autoantibodies in serum of patients with cystic fibrosis. Scand J Respir Dis 1975;56:38-46.

10 Schiotz PO, Hoiby N, Permin H, et al. $\lg A$ and $\lg G$ antibodies against surface antigens of Pseudomonas aeruginosa in sputum and serum from patients with cystic fibrosis. Acta Pathol Microbiol Scand [C ] 1979;87C:229-33.

11 Klinger JD, Straus DC, Hilton CB, et al. Antibodies to proteases and exotoxin A of Pseudomonas aeruginosa in patients with cystic fibrosis: demonstration by radioimmunoassay. J Infect Dis 1978;138:49-8.

12 Jagger KS, Robinson DL, Franz MN, et al. Detection by enzyme-linked immunosorbent assays of antibody specific for Pseudomonas proteases and exotoxin $A$ in sera from cystic fibrosis patients. J Clin Microbiol 1982;15:1054-8.

13 Brett MM, Ghoneim AT, Littlewood JM. Serum antibodies to Pseudomonas aeruginosa in cystic fibrosis. Arch Dis Child 1986;61:1114-20.

14 Brett MM, Ghoneim AT, Littlewood JM. Prediction and diagnosis of early Pseudomonas aeruginosa infection in cystic fibrosis: a follow-up study. J Clin Microbiol 1988;26:1565-70.

15 Brett MM, Ghoneim AT, Littlewood JM. Serum IgA antibodies against Pseudomonas aeruginosa in cystic fibrosis. Arch Dis Child 1990;65:259-63.

16 Brett MM, Simmonds EJ, Ghoneim AT, et al. The value of serum IgG titres against Pseudomonas aeruginosa in the management of early pseudomonal infection in cystic fibrosis. Arch Dis Child 1992;67:1086-8.

17 Burns JL, Gibson RL, McNamara S, et al. Longitudinal assessment of Pseudomonas aeruginosa in young children with cystic fibrosis. J Infect Dis $2001 ; 183: 444-52$.

18 Doring G, Hoiby N. Longitudinal study of immune response to Pseudomonas aeruginosa antigens in cystic fibrosis. Infect Immun 1983;42:197-201.

19 Hollsing AE, Granstrom M, Vasil ML, et al. Prospective study of serum antibodies to Pseudomonas aeruginosa exoproteins in cystic fibrosis. J Clin Microbiol 1987;25:1868-74.

20 Prader A, Largo RH, Molinari L, et al. Physical growth of Swiss children from birth to 20 years of age. First Zurich longitudinal study of growth and development. Helv Paediatr Acta Suppl 1989;52:1-125.

21 Bland JM, Altman DG. Statistical methods for assessing agreement between two methods of clinical measurement. Lancet 1986;1:307-10.

22 Jones AM, Dodd ME, Govan JR, et al. Prospective surveillance for Pseudomonas aeruginosa cross-infection at a cystic fibrosis center. Am J Respir Crit Care Med 2005;171:257-60.

23 Doring G, Conway SP, Heijerman HG, et al. Antibiotic therapy against Pseudomonas aeruginosa in cystic fibrosis: a European consensus. Eur Respir J 2000;16:749-67.

24 Kabra SK, Alok A, Kapil A, et al. Can throat swab after physiotherapy replace sputum for identification of microbial pathogens in children with cystic fibrosis? Indian J Pediatr 2004;71:21-3.

25 Ramsey BW, Wentz KR, Smith AL, et al. Predictive value of oropharyngeal cultures for identifying lower airway bacteria in cystic fibrosis patients. Am Rev Respir Dis 1991; 144:331-7.

26 Armstrong DS, Grimwood K, Carlin JB, et al. Bronchoalveolar lavage or oropharyngeal cultures to identify lower respiratory pathogens in infants with cystic fibrosis. Pediatr Pulmonol 1996;21:267-75.

27 Rosenfeld M, Emerson J, Accurso F, et al. Diagnostic accuracy of oropharyngeal cultures in infants and young children with cystic fibrosis. Pediatr Pulmonol 1999;28:321-8.

28 Bavernfeind A, Przyklenk B. Microbiological background for antiPseudomonas aeruginosa vaccination in cystic fibrosis. Behring Inst Mitt 1997;98:256-61

29 Emerson J, Rosenfeld M, McNamara S, et al. Pseudomonas aeruginosa and other predictors of mortality and morbidity in young children with cystic fibrosis. Pediatr Pulmonol 2002;34:91-100. 